\title{
Analytic-Numerical Solution of Random Boundary Value Heat Problems in a Semi-Infinite Bar
}

\author{
M.-C. Casabán, J.-C. Cortés, B. García-Mora, and L. Jódar \\ Instituto Universitario de Matemática Multidisciplinar, Building $8 G$ Access C, 2nd Floor, Universitat Politècnica de València, \\ Camino de Vera s/n, 46022 Valencia, Spain
}

Correspondence should be addressed to M.-C. Casabán; macabar@imm.upv.es

Received 9 July 2013; Accepted 27 August 2013

Academic Editor: Benito Chen-Charpentier

Copyright (C) 2013 M.-C. Casabán et al. This is an open access article distributed under the Creative Commons Attribution License, which permits unrestricted use, distribution, and reproduction in any medium, provided the original work is properly cited.

\begin{abstract}
This paper deals with the analytic-numerical solution of random heat problems for the temperature distribution in a semi-infinite bar with different boundary value conditions. We apply a random Fourier sine and cosine transform mean square approach. Random operational mean square calculus is developed for the introduced transforms. Using previous results about random ordinary differential equations, a closed form solution stochastic process is firstly obtained. Then, expectation and variance are computed. Illustrative numerical examples are included.
\end{abstract}

\section{Introduction}

Temperature and heat flow are two important quantities in the problem of heat conduction. Temperature at any point of a solid is completely determined by its numerical value because it is a scalar quantity, whereas heat flow is defined by its value and direction, and it depends on the properties of the material that are neither uniform nor pure [1]. These uncertainties about material apart from measurement error deserve a random approach. Random heat transfer in a finite medium has been treated in [2] by developing a random perturbation method, in [3] by using finite element method, and in $[4,5]$ using finite difference methods.

It is well known that the integral transform technique to solve deterministic heat problems is very powerful and efficient because, by the combined use of the direct and inverse transform, the problem is simplified, by transforming partial differential equations into ordinary differential ones. In this paper, we introduce the random Fourier sine and cosine transforms and their mean square operational calculus to solve random temperature distribution in a semi-infinite bar with random temperature or random heat flow at one end. Using results about random ordinary differential equations of [6] allows us to find closed-form solutions stochastic process (s.p.) of random heat problems as in the deterministic case.

Although some authors deal with the uncertainty by using the Brownian motion and the Itô calculus $[7,8]$, we use a random mean square approach (m.s.) for two main reasons. The first one is that the m.s. solution coincides with the deterministic solution when data are deterministic. The second one is that if $V(x, t)$ is an m.s. approximation of $u(x, t)$, then the expectation and the variance of $V(x, t)$ converge to the expectation and variance of $u(x, t)$, respectively; see [9, chapter 4].

This paper is organized as follows. In Section 2, some preliminaries about m.s. calculus definitions, properties, and results are included. In Section 3, the random Fourier sine and cosine transforms are introduced and important m.s. operational rules related to the random Fourier sine (cosine) transform of a s.p. and of its m.s. derivatives, that is, the key to solve partial differential equations in terms of ordinary differential equations, are given. Sections 4 and 5 deal with random boundary value problems associated with the random heat equation

$$
u_{t}(x, t)=L u_{x x}(x, t), \quad x>0, t>0,
$$

where $L$ is a positive random variable (r.v.) whose properties will be specified later. For the deterministic case, the boundary conditions of these problems are called of third and second kind, respectively [1, page 52]. In Section 6, some illustrative examples are studied and in Section 7 a set of conclusions are given. 


\section{Preliminaries about Random Mean Square Calculus}

In this section, we review some important concepts, definitions, and results related to the random $L_{p}$ calculus, mainly focusing on the mean square (m.s.) and mean fourth (m.f.) calculus, which correspond to $p=2$ and $p=4$, respectively (see [10] for further details). After that, a relevant class of r.v.'s that will play an important role in the development of next sections is studied.

Let $(\Omega, \mathscr{F}, \mathscr{P})$ be a probabilistic space. Let $p \geq 1$ be a real number. A real r.v. $U$ defined on $(\Omega, \mathscr{F}, \mathscr{P})$ is called of order $p$, if

$$
\mathrm{E}\left[|U|^{p}\right]<+\infty
$$

where $\mathrm{E}[\cdot]$ denotes the expectation operator. The space $L_{p}$ of all the real r.v.s of order $p$, endowed with the norm

$$
\|U\|_{p}=\left(\mathrm{E}\left[|U|^{p}\right]\right)^{1 / p}
$$

is a Banach space, [11, page 9].

Let $\left\{U_{n}: n \geq 0\right\}$ be a sequence of r.v.s of order $p$. We say that it is convergent in the $p$ th mean to the real r.v. $U \in L_{p}$, if

$$
\lim _{n \rightarrow+\infty}\left\|U_{n}-U\right\|_{p}=0
$$

This type of convergence is often expressed by $\lim _{n \rightarrow \infty} U_{n}=$ $U$. The symbol l.i.m. denotes the limit in the $p$ th mean. If $p_{2} \geq p_{1}$, then $L_{p_{2}} \subseteq L_{p_{1}}$. In addition, if $\left\{U_{n}: n \geq 0\right\}$ is $p_{2}$ th mean convergent to $U \in L_{p_{2}}$, then $\left\{U_{n}: n \geq 0\right\}$ is also $p_{1}$ th mean convergent to $U \in L_{p_{1}}$ [11, page 13]. Convergences in $L_{2}$ and $L_{4}$ are usually referred to as m.s. and m.f. convergence, respectively. If $\left\{U_{n}: n \geq 0\right\}$ is a sequence of 2-r.v's in $L_{2}$ m.s. convergent to $U \in L_{2}$, then from Theorem 4.3.1 of [9, page 88 ] one gets

$$
\lim _{n \rightarrow \infty} \mathrm{E}\left[U_{n}\right]=\mathrm{E}[U], \quad \lim _{n \rightarrow \infty} \operatorname{Var}\left[U_{n}\right]=\operatorname{Var}[U],
$$

where $\operatorname{Var}[\cdot]$ denotes the variance operator.

Let $T$ be a subset of the real line. A family $\{U(t): t \in T\}$ of real r.v.s of order $p$ is said to be a s.p. of order $p$ or, in short, a $p$-s.p. if

$$
\mathrm{E}\left[|U(t)|^{p}\right]<+\infty, \quad \forall t \in T .
$$

We say that $\{U(t): t \in T\}$ is $p$ th mean continuous at $t \in T$, if

$$
\|U(t+h)-U(t)\|_{p} \longrightarrow 0 \quad \text { as } h \longrightarrow 0, t, t+h \in T .
$$
that

Furthermore, if there exists a s.p. $U^{\prime}(t)$ of order $p$, such

$$
\begin{array}{r}
\left\|\frac{U(t+h)-U(t)}{h}-U^{\prime}(t)\right\|_{p} \longrightarrow 0, \\
\text { as } h \longrightarrow 0, t, t+h \in T,
\end{array}
$$

then we say that $\{U(t): t \in T\}$ is $p$ th mean differentiable at $t \in T$ and $U^{\prime}(t)$ is the $p$-derivative of $U(t)$.
In the particular cases that $p=2,4$, aforementioned definitions lead to the corresponding concepts of mean square (m.s.) and mean fourth (m.f.) continuity and differentiability. Furthermore, it is easy to establish by the Schwarz's inequality that $\|U V\|_{2} \leq\|U\|_{4}\|V\|_{4}$ (see [10]), which prove that m.f. continuity and differentiability entail m.s. continuity and differentiability, respectively.

In accordance with [9, page 99], [12], we say that a s.p. $\{V(x): x \in \mathbb{R}\}$ with $V(x) \in L_{p}$ for all $x$, is locally integrable in $\mathbb{R}$ if, for all finite interval $[a, b] \subset \mathbb{R}$, the integral

$$
\int_{a}^{b} V(x) d x
$$

exits in $L_{p}$. We say that $\{V(x): x \in \mathbb{R}\}$ is absolutely integrable in $L_{p}$, if

$$
\int_{-\infty}^{+\infty}\|V(x)\|_{p} d x<+\infty .
$$

Now, we introduce an important type of r.v.s, $L$, that have played a significant role in the m.s. solution of random ordinary differential equations (see [13] and references therein), and which will be used later. We will assume that such r.v.s $L$ have absolute moments with respect to the origin that increases at the most exponentially; that is, there exist a nonnegative integer $n_{0}$ and positive constants $M$ and $H$ such that

$$
\mathrm{E}\left[|L|^{n}\right] \leq M H^{n}, \quad \forall n \geq n_{0}, \quad \text { that is, } \mathrm{E}\left[|L|^{n}\right]=\mathcal{O}\left(H^{n}\right) .
$$

From (11) and definition (3) for $p=2$, for each $x \in \mathbb{R}$, one gets

$$
\begin{aligned}
\left(\left\|e^{-L x^{2}}\right\|_{2}\right)^{2} & =\mathrm{E}\left[e^{\left.-2 L x^{2}\right]}=\mathrm{E}\left[\sum_{n \geq 0} \frac{(-2)^{n} L^{n} x^{2 n}}{n !}\right]\right. \\
& =\sum_{n \geq 0} \frac{(-2)^{n} x^{2 n} \mathrm{E}\left[L^{n}\right]}{n !} \\
& \leq \sum_{n \geq 0} \frac{(-2)^{n} x^{2 n} \mathrm{E}\left[|L|^{n}\right]}{n !} \\
& \leq M \sum_{n \geq 0} \frac{(-2)^{n} x^{2 n} H^{n}}{n !}=M e^{-2 H x^{2}}, \quad M>0 .
\end{aligned}
$$

Thus,

$$
\left\|e^{-L x^{2}}\right\|_{2} \leq \widetilde{M} e^{-H x^{2}}, \quad x \in \mathbb{R}, \widetilde{M}=\sqrt{M}>0 .
$$

Remark 1. The lack of explicit formulae for the absolute moments with respect to the origin of some standard r.v's as well as the aim of looking for a general approach to deal with the widest range of random inputs, we are going to take advantage of censuring method (see [14, chapter 5]) to show that truncated r.v.s satisfy condition (11). Let us assume a r.v. $L$ that satisfies

$$
l_{1} \leq l=L(\omega) \leq l_{2}, \quad \forall \omega \in \Omega .
$$


Then,

$$
\mathrm{E}\left[|L|^{n}\right]=\int_{l_{1}}^{l_{2}}|l|^{n} f_{L}(l) d l \leq H^{n},
$$

where $f_{L}(l)$ denotes the probability density function (p.d.f.) of r.v. $L$ and $H=\max \left(\left|l_{1}\right|,\left|l_{2}\right|\right)$. Indeed, in the case that $H>1$, one gets

$$
\int_{l_{1}}^{l_{2}}|l|^{n} f_{L}(l) d l \leq H^{n} \int_{l_{1}}^{l_{2}} f_{L}(l) d l=H^{n} .
$$

Notice that, in the last step, we have applied that the integral of the right-hand side is just 1 because $f_{L}(l)$ is a p.d.f. The other cases can be analyzed analogously. Substituting the integral by a sum in (15), previous reasoning remains true when $L$ is a discrete r.v. As a consequence, important r.v.s such as binomial, hypergeometric, uniform, or beta satisfy condition (11) related to the absolute moments of $L$. Although many other unbounded r.v.s can also verify condition (11), we do not need to check it each case; since censuring their codomain suitably, we are legitimated to approximate them. Hence, truncations of r.v.s such as exponential or Gaussian satisfy condition (11). The larger the censured interval is, the better the approximations are. However, in practice, intervals relatively short provide very good approximations. For instance, as an illustrative example, notice that the truncated interval $[\mu-3 \sigma, \mu+3 \sigma]$ contains the $99.7 \%$ of the probability mass of a Gaussian r.v. with mean $\mu$ and standard deviation $\sigma>0$.

For the sake of clarity in the presentation, we state the m.s. differentiation of integrals whose proof is a direct consequence of the deterministic case [12, page 99] and the m.s. differentiation theorem for a sequence of 2-s.p. [15].

Lemma 2 (m.s. differentiation of infinite integrals). Let $g(x, t)$ be a 2-s.p. m.s. continuous with m.s. continuous partial derivative $\partial g(x, t) / \partial t$. Assume the following hypotheses.

(i) $G(t)=\int_{a}^{+\infty} g(x, t) d x$ is m.s. pointwise convergent for each $t>0$.

(ii) $\int_{a}^{+\infty}(\partial g(x, t) / \partial t) d x$ is m.s. uniformly convergent in $[t-\delta, t+\delta], \delta>0$ for each $t>0$.

Then, the process $G(t)$ is m.s. differentiable, and

$$
G^{\prime}(t)=\int_{a}^{+\infty} \frac{\partial g(x, t)}{\partial t} d x .
$$

\section{Random Fourier Sine and Cosine Transforms' Operational Calculus}

We begin this section by introducing the definition of the random Fourier sine and cosine transforms of a 2-s.p. $\{u(x)$ : $x>0\}$ m.s. locally integrable, and m.s. absolutely integrable; that is,

$$
\int_{0}^{\infty}\|u(x)\|_{2} d x<+\infty
$$

as the s.p.s

$$
\begin{aligned}
& \mathfrak{F}_{\mathfrak{\xi}}[u(x)](\xi)=\int_{0}^{\infty} u(x) \sin (\xi x) d x, \quad \xi>0, \\
& \mathfrak{F}_{\mathfrak{c}}[u(x)](\xi)=\int_{0}^{\infty} u(x) \cos (\xi x) d x, \quad \xi>0,
\end{aligned}
$$

respectively. Note that from (18) both integrals appearing in (19) and (20) are convergent in $L_{2}$ and thus they are 2-s.p.s well defined.

Following the ideas of the deterministic inverse Fourier sine and cosine transforms, [16, chapter 2$]$, we define the random inverse Fourier sine and cosine transforms of a 2s.p. $F(\xi)$ m.s. locally and m.s. absolutely integrable by the formulae

$$
\begin{array}{ll}
\mathfrak{F}_{\mathfrak{g}}^{-1}[F(\xi)](x)=\frac{2}{\pi} \int_{0}^{\infty} F(\xi) \sin (\xi x) d \xi, & x>0, \\
\mathfrak{F}_{\mathfrak{c}}^{-1}[F(\xi)](x)=\frac{2}{\pi} \int_{0}^{\infty} F(\xi) \cos (\xi x) d \xi, & x>0,
\end{array}
$$

respectively.

The following result contains some m.s. operational rules that will be used in Sections 4 and 5 to solve random heat problems in a semi-infinite medium.

Theorem 3. Let $\{u(x): x>0\}$ be a 2-s.p. twice m.s. differentiable with $u^{\prime \prime}(x)$ m.s. locally integrable, and with $u(x)$, $u^{\prime}(x)$, and $u^{\prime \prime}(x)$ m.s. absolutely integrable in $[0, \infty[$. Then,

(i) $\mathfrak{\mho}_{\mathfrak{g}}\left[u^{\prime}(x)\right](\xi)=-\xi \mathfrak{\mho}_{\mathfrak{c}}[u(x)](\xi)$,

$$
\xi>0,
$$

(ii) $\mathfrak{F}_{\mathfrak{c}}\left[u^{\prime}(x)\right](\xi)=-u(0)+\xi \mathfrak{F}_{\mathfrak{g}}[u(x)](\xi)$,

$$
\xi>0,
$$

(iii) $\mathfrak{\mho}_{\mathfrak{\xi}}\left[u^{\prime \prime}(x)\right](\xi)=u(0) \xi-\xi^{2} \mathfrak{\mho}_{\mathfrak{\xi}}[u(x)](\xi)$,

$$
\xi>0,
$$

(iv) $\mathfrak{\mho}_{\mathfrak{c}}\left[u^{\prime \prime}(x)\right](\xi)=-u^{\prime}(0)-\xi^{2} \mathfrak{\mho}_{\mathfrak{c}}[u(x)](\xi)$,

$$
\xi>0 .
$$

Proof. We present the proof of each formula separately.

(i) By the rule for the m.s. derivative of a product of a 2s.p. by a deterministic function, [9, page 96], it follows that

$$
(u(x) \sin (\xi x))^{\prime}=u^{\prime}(x) \sin (\xi x)+\xi u(x) \cos (\xi x),
$$

or

$$
u^{\prime}(x) \sin (\xi x)=(u(x) \sin (\xi x))^{\prime}-\xi u(x) \cos (\xi x) .
$$


From definitions (19) and (28), one gets

$$
\begin{aligned}
\mathfrak{\mho}_{\mathfrak{\xi}}\left[u^{\prime}(x)\right](\xi)= & \int_{0}^{\infty} u^{\prime}(x) \sin (\xi x) d x \\
= & \int_{0}^{\infty}(u(x) \sin (\xi x))^{\prime} d x \\
& -\xi \int_{0}^{\infty} u(x) \cos (\xi x) d x .
\end{aligned}
$$

From the fundamental theorem of the m.s. calculus [9, page 104], we have

$$
\int_{0}^{R} u^{\prime}(x) d x=u(R)-u(0)
$$

From (30), as $u^{\prime}(x)$ is m.s. absolutely integrable, it follows that

$$
\begin{aligned}
\int_{0}^{\infty} u^{\prime}(x) d x & =\underset{R \rightarrow \infty}{\lim .{ }_{0}} \int_{0}^{R} u^{\prime}(x) d x \\
& =\left(\lim _{R \rightarrow \infty} u(R)\right)-u(0) .
\end{aligned}
$$

Hence, the limit $\ell=\lim _{\cdot R \rightarrow \infty} u(R)$ is finite. Furthermore, as $u(x)$ is m.s. absolutely integrable, by the Cauchy condition of the integral

$$
\int_{0}^{\infty}\|u(x)\|_{2} d x
$$

one gets that

$$
\underset{R \rightarrow \infty}{\lim } u(R)=0 \text {. }
$$

By the fundamental theorem of the m.s. calculus [9, page 104], we also have

$$
\int_{0}^{R}(u(x) \sin (\xi x))^{\prime} d x=u(R) \sin (\xi R), \quad \xi>0 .
$$

From (33) and (34) one gets that

$$
\begin{aligned}
\int_{0}^{\infty}(u(x) \sin (\xi x))^{\prime} d x \\
\quad=\lim _{R \rightarrow \infty} \int_{0}^{R}(u(x) \sin (\xi x))^{\prime} d x=0 .
\end{aligned}
$$

Finally, from (29) and (35) and definition (20), one gets the proof of (i).

(ii) By the rule for the m.s. derivative of a product of a 2s.p. by a deterministic function $[9$, page 96$]$ we have

$$
(u(x) \cos (\xi x))^{\prime}=u^{\prime}(x) \cos (\xi x)-\xi u(x) \sin (\xi x),
$$

or

$$
u^{\prime}(x) \cos (\xi x)=(u(x) \cos (\xi x))^{\prime}+\xi u(x) \sin (\xi x) .
$$

Now, by definition (20) applied to $u^{\prime}(x)$ and (37), it follows that

$$
\begin{aligned}
\mathfrak{F}_{\mathfrak{c}}\left[u^{\prime}(x)\right](\xi)= & \int_{0}^{\infty} u^{\prime}(x) \cos (\xi x) d x \\
= & \int_{0}^{\infty}(u(x) \cos (\xi x))^{\prime} d x \\
& +\xi \int_{0}^{\infty} u(x) \sin (\xi x) d x .
\end{aligned}
$$

By the fundamental theorem of the m.s. calculus [9, page 104],

$$
\int_{0}^{\infty}(u(x) \cos (\xi x))^{\prime} d x=\underset{R \rightarrow \infty}{\lim . m}\{u(R) \cos (\xi R)-u(0)\} .
$$

As in the proof of part (i), l.i.m. . $_{R \rightarrow \infty} u(R)=0$, and thus from (39) one gets

$$
\int_{0}^{\infty}(u(x) \cos (\xi x))^{\prime} d x=-u(0) .
$$

From (38) and (40), one gets (24).

(iii) By applying part (i) to $u^{\prime}(x)$, it follows directly (25).

(iv) It is a direct consequence of the application of part (ii) to $u^{\prime}(x)$.

\section{Random Heat Problem with Third Kind Boundary Condition}

In this section, we deal with the random heat problem for the temperature distribution $u(x, t)$ in a semi-infinite bar with random temperature at the end $x=0$ and zero initial temperature

$$
\begin{gathered}
u_{t}(x, t)=L u_{x x}(x, t), \quad x>0, t>0, \\
u(0, t)=A, \quad t>0, \\
u(x, 0)=0, \quad x>0,
\end{gathered}
$$

where $L$ and $A$ both are independent positive 4-r.v.s, satisfying properties to be specified later. Assume that problem (41)-(43) admits a solution 2-s.p. $u(x, t)$ m.s. locally and m.s. absolutely integrable, and let us denote

$$
\mathfrak{F}_{\mathfrak{g}}[u(\cdot, t)](\xi)=\mathscr{U}(t)(\xi), \quad \xi>0,
$$

what means that $u(x, t)$ is regarded as a process of the active variable $x$, for fixed $t>0$. By applying the random Fourier sine transform to both members of (41) and properties of Theorem 3, it follows that

$$
\begin{aligned}
\mathfrak{\Im}_{\mathfrak{s}}\left[u_{x x}(\cdot, t)\right](\xi) & =\xi u(0, t)-\xi^{2} \mathfrak{\mho}_{\mathfrak{s}}[u(\cdot, t)](\xi) \\
& =A \xi-\xi^{2} \mathcal{U}(t)(\xi),
\end{aligned}
$$

and from Lemma 2,

$$
\mathfrak{\mho}_{\mathfrak{\xi}}\left[u_{t}(\cdot, t)\right](\xi)=\frac{d}{d t}(\mathcal{U}(t)(\xi)) .
$$

From condition (43), it follows that

$$
\mathfrak{\mho}_{\mathfrak{\xi}}[u(\cdot, 0)](\xi)=\mathscr{U}(0)(\xi)=0 .
$$


Hence, the transformed problem becomes the following random initial value problem for the variable $t$ :

$$
\begin{gathered}
\frac{d}{d t}(\mathcal{U}(t)(\xi))+L \xi^{2} \mathcal{U}(t)(\xi)=\xi A L, \quad t>0, \\
\mathcal{U}(0)(\xi)=0 .
\end{gathered}
$$

Let us assume that

$\Phi_{L}(t)=\mathrm{E}\left[e^{t L}\right]$ is locally bounded about $t=0$,

being $\Phi_{L}(t)$ the moment generating function of r.v. $L$. Then, by Theorem 8 of [6], the solution s.p. of problem (48) is given by

$$
\mathcal{U}(t)(\xi)=\frac{A}{\xi}-\frac{A}{\xi} e^{-t \xi^{2} L}
$$

By using the random inverse Fourier sine transform (21), one gets

$$
u(x, t)=\mathfrak{F}_{\mathfrak{J}}^{-1}[\mathcal{U}(t)(\xi)]=\frac{2 A}{\pi}\left\{\mathrm{I}_{1}(x)-\mathrm{I}_{2}(x, t)\right\},
$$

where

$$
\begin{gathered}
\mathrm{I}_{1}(x)=\int_{0}^{\infty} \frac{\sin (\xi x)}{\xi} d \xi, \\
\mathrm{I}_{2}(x, t)=\int_{0}^{\infty} e^{-t \xi^{2} L} \frac{\sin (\xi x)}{\xi} d \xi .
\end{gathered}
$$

Putting the substitution $\xi x=\tau$, we have

$$
\mathrm{I}_{1}(x)=\int_{0}^{\infty} \frac{\sin (\tau)}{\tau} d \tau=\frac{\pi}{2},
$$

and dealing with $\mathrm{I}_{2}(x, t)$, we have

$$
\mathrm{I}_{2}(x, t)=\int_{0}^{\infty} e^{-t\left(\tau^{2} / x^{2}\right) L} \frac{\sin (\tau)}{\tau} d \tau .
$$

We start paying attention to the derivative with respect to the variable $x$ of the s.p. $\mathrm{I}_{2}(x, t)$. Note that, under condition (11), the property (13) holds:

$$
\left\|e^{-t L \xi^{2}}\right\|_{2} \leq \widetilde{M} e^{-H t \xi^{2}}, \quad t>0
$$

for some positive constants $\widetilde{M}$ and $H$. Hence, it is easy to check that $\mathrm{I}_{2}(x, t)$ is m.s. uniformly convergent in a neighbourhood $\left[x_{0}-\delta, x_{0}+\delta\right], \delta>0$, of each $x_{0}>0, t>0$.

Note also that, from (55), the integral of the derivative with respect to $x$ of the integrand of $\mathrm{I}_{2}(x, t)$ (see (52))

$$
J(x, t)=\int_{0}^{\infty} e^{-t \xi^{2} L} \cos (\xi x) d \xi
$$

is m.s. absolutely uniformly convergent in a neighbourhood $\left[x_{0}-\delta, x_{0}+\delta\right], \delta>0$, of each $x_{0}>0, t>0$. Hence, for each $x>0, t>0,(56)$ defines a 2-r.v. By Lemma 2, it follows that

$$
\frac{\partial \mathrm{I}_{2}(x, t)}{\partial x}=J(x, t)=\int_{0}^{\infty} e^{-t \xi^{2} L} \cos (\xi x) d \xi .
$$

By [12, page 61], fixed $x>0, t>0$, each realization of (56), denoted by $J(x, t)(\omega)$, can be computed as follows:

$$
\begin{aligned}
J(x, t)(\omega)= & \frac{1}{2} \sqrt{\frac{\pi}{t L(\omega)}} e^{-x^{2} / 4 t L(\omega)}, \\
& x>0, t>0, \omega \in \Omega .
\end{aligned}
$$

Thus,

$$
J(x, t)=\frac{1}{2} \sqrt{\frac{\pi}{t L}} e^{-x^{2} / 4 t L}, \quad x>0, t>0 .
$$

By $(52) \mathrm{I}_{2}(0, t)=0$, the fundamental theorem of the m.s. calculus [9, page 104] yields

$$
\begin{aligned}
\mathrm{I}_{2}(x, t) & =\mathrm{I}_{2}(0, t)+\int_{0}^{x} \frac{\partial \mathrm{I}_{2}(s, t)}{\partial s} d s \\
& =\frac{1}{2} \sqrt{\frac{\pi}{t L}} \int_{0}^{x} e^{-s^{2} / 4 t L} d s .
\end{aligned}
$$

From (51), (53), and (60), one gets

$$
u(x, t)=A\left(1-\frac{1}{\sqrt{\pi t L}} \int_{0}^{x} e^{-s^{2} / 4 t L} d s\right) .
$$

Using the independence of r.v.s $A$ and $L$, one gets that the expectation and the variance function of the solution s.p. are, respectively,

$$
\begin{array}{r}
\mathrm{E}[u(x, t)]=\mathrm{E}[A]\left(1-\frac{1}{\sqrt{\pi t}} \int_{0}^{x} \mathrm{E}\left[\frac{1}{\sqrt{L}} e^{-s^{2} / 4 t L}\right] d s\right), \\
x>0, t>0,
\end{array}
$$

$$
\operatorname{Var}[u(x, t)]=\mathrm{E}\left[(u(x, t))^{2}\right]-(\mathrm{E}[u(x, t)])^{2},
$$

where

$$
\begin{aligned}
\mathrm{E}\left[(u(x, t))^{2}\right]= & \mathrm{E}\left[A^{2}\right] \\
& \times\left(1-\frac{2}{\sqrt{\pi t}} \int_{0}^{x} \mathrm{E}\left[\frac{1}{\sqrt{L}} e^{-s^{2} / 4 t L}\right] d s+\frac{1}{\pi t}\right. \\
& \left.\times \iint_{0}^{x} \mathrm{E}\left[\frac{1}{L} e^{-\left(s_{1}^{2}+s_{2}^{2}\right) / 4 t L}\right] d s_{1} d s_{2}\right)
\end{aligned}
$$

Summarizing, the following result has been established.

Theorem 4. Let us consider the random heat problem given by (41)-(43) where $L$ and $A$ are independent positive 4-r.v.s. Assume that $L$ also satisfies conditions (11) and (49). Then, the solution stochastic process $u(x, t)$ of this problem is given by (61). In addition, (62)-(64) are closed expressions for its expectation and variance.

\section{Random Heat Problem with Second Kind Boundary Condition}

This section deals with the random heat problem for the temperature distribution $u(x, t)$ in a semi-infinite bar with 
zero initial temperature and where the heat flow $u_{x}(0, t)$ at the end $x=0$ is given by s.p. $g(t ; B)$ :

$$
\begin{gathered}
u_{t}(x, t)=L u_{x x}(x, t), \quad x>0, t>0, \\
u_{x}(0, t)=g(t ; B), \quad t>0 \\
u(x, 0)=0, \quad x \geq 0
\end{gathered}
$$

where $L$ and $B$ both are independent 4-r.v.s and $L$ is positive and satisfies properties (11) and (49). We also assume that $g(t ; B)$ is m.f. continuous. Assuming that problem (65)-(67) admits a solution 2-s.p. $u(x, t)$ locally and absolutely m.s. integrable, let us denote

$$
\mathfrak{\mho}_{\mathfrak{c}}[u(\cdot, t)](\xi)=\mathscr{U}(t)(\xi), \quad \xi>0,
$$

which means that $u(x, t)$ is regarded as a process of the active variable $x$, for fixed $t>0$. By applying the random Fourier cosine transform to both members of (65) and properties of Theorem 3, one gets

$$
\begin{aligned}
\mathfrak{F}_{\mathfrak{c}}\left[u_{x x}(\cdot, t)\right](\xi) & =-u_{x}(0, t)-\xi^{2} \mathfrak{F}_{\mathfrak{c}}[u(\cdot, t)](\xi) \\
& =-g(t ; B)-\xi^{2} \mathcal{U}(t)(\xi)
\end{aligned}
$$

In addition, from (68) and (67), one gets

$$
\mathfrak{F}_{\mathfrak{c}}[u(\cdot, 0)](\xi)=\mathscr{U}(0)(\xi)=0 .
$$

By Lemma 2, we also have

$$
\mathfrak{F}_{\mathfrak{c}}\left[u_{t}(\cdot, t)\right](\xi)=\frac{d}{d t}(\mathcal{U}(t)(\xi))
$$

Thus, the transformed random ordinary initial value problem in the variable $t$ becomes

$$
\begin{gathered}
\frac{d}{d t}(\mathcal{U}(t)(\xi))+L \xi^{2} \mathcal{U}(t)(\xi) \\
=-\operatorname{Lg}(t ; B), \quad t>0, \\
\mathcal{U}(0)(\xi)=0 .
\end{gathered}
$$
by

By using Theorem 8 of [6], the solution of (72) is given

$$
\mathcal{U}(t)(\xi)=-L e^{-t \xi^{2} L} \int_{0}^{t} g(s ; B) e^{s \xi^{2} L} d s, \quad t>0
$$

Taking in (73) the random inverse Fourier cosine transform (22) and using Fubini theorem in normed spaces [17, page 175], it follows that

$$
\begin{aligned}
u(x, t) & =\mathfrak{F}_{\mathfrak{c}}^{-1}[\mathcal{U}(t)(\xi)] \\
& =-\frac{2 L}{\pi} \int_{0}^{\infty} e^{-t \xi^{2} L} \int_{0}^{t} g(s ; B) e^{s \xi^{2} L} \cos (\xi x) d s d \xi \\
& =-\frac{2 L}{\pi} \int_{0}^{t} g(s ; B)\left\{\int_{0}^{\infty} e^{-\xi^{2}(t-s) L} \cos (\xi x) d \xi\right\} d s .
\end{aligned}
$$

With the notation of (56), we have

$$
u(x, t)=-\frac{2 L}{\pi} \int_{0}^{t} g(s ; B) J(x, t-s) d s, \quad x>0, t>0 .
$$

For the sake of convenience, let us write

$$
\begin{aligned}
J(x, t-s) & =\int_{0}^{\infty} e^{-\xi^{2}(t-s) L} \cos (\xi x) d \xi \\
& =[\xi \sqrt{t-s}=\nu] \\
& =\frac{1}{\sqrt{t-s}} \int_{0}^{\infty} e^{-\nu^{2} L} \cos \left(\frac{v x}{\sqrt{t-s}}\right) d \nu .
\end{aligned}
$$

Note that under condition (11), the random integral appearing in (76) is m.s. absolutely uniformly convergent in a neighbourhood $\left[x_{0}-\delta, x_{0}+\delta\right], \delta>0$, of each $x_{0}>0, t>0$. Furthermore, from [12, page 61] each realization

$$
\begin{aligned}
& \int_{0}^{\infty} e^{-v^{2} L(\omega)} \cos \left(\frac{v x}{\sqrt{t-s}}\right) d v \\
& =\frac{1}{2} \sqrt{\frac{\pi}{(t-s) L(\omega)}} e^{-x^{2} / 4(t-s) L(\omega)}, \quad \omega \in \Omega .
\end{aligned}
$$

Hence, from (76)-(77), one gets

$$
J(x, t-s)=\frac{1}{2} \sqrt{\frac{\pi}{(t-s) L}} e^{-x^{2} / 4(t-s) L},
$$

and from (75) it follows that

$$
\begin{aligned}
u(x, t) & =-\sqrt{\frac{L}{\pi}} \int_{0}^{t} \frac{g(s ; B)}{\sqrt{t-s}} e^{-x^{2} / 4(t-s) L} d s \\
& =[\sqrt{t-s}=v] \\
& =2 \sqrt{\frac{L}{\pi}} \int_{0}^{\sqrt{t}} g\left(t-v^{2} ; B\right) e^{-(x / 2 v \sqrt{L})^{2}} d v, \\
x>0, t & >0 .
\end{aligned}
$$

Using the independence of r.v.s $B$ and $L$, one computes the expectation of the solution s.p. $u(x, t)$ :

$$
\begin{aligned}
\mathrm{E}[u(x, t)]=\frac{2}{\sqrt{\pi}} \int_{0}^{\sqrt{t}} \mathrm{E}\left[g\left(t-v^{2} ; B\right)\right] \\
\times \mathrm{E}\left[\sqrt{L} e^{-(x / 2 v \sqrt{L})^{2}}\right] d v .
\end{aligned}
$$

Taking into account (63), the variance of $u(x, t)$ is computed from (80) and

$$
\begin{aligned}
& \mathrm{E}\left[(u(x, t))^{2}\right] \\
& =\frac{4}{\pi} \iint_{0}^{\sqrt{t}} \mathrm{E}\left[g\left(t-v_{1}^{2} ; B\right) g\left(t-v_{2}^{2} ; B\right)\right] \\
& \quad \times \mathrm{E}\left[L e^{-\left(x / 2 v_{1} v_{2} \sqrt{L}\right)^{2}\left(v_{1}^{2}+v_{2}^{2}\right)}\right] d v_{1} d v_{2} .
\end{aligned}
$$

Summarizing, the following result has been established. 


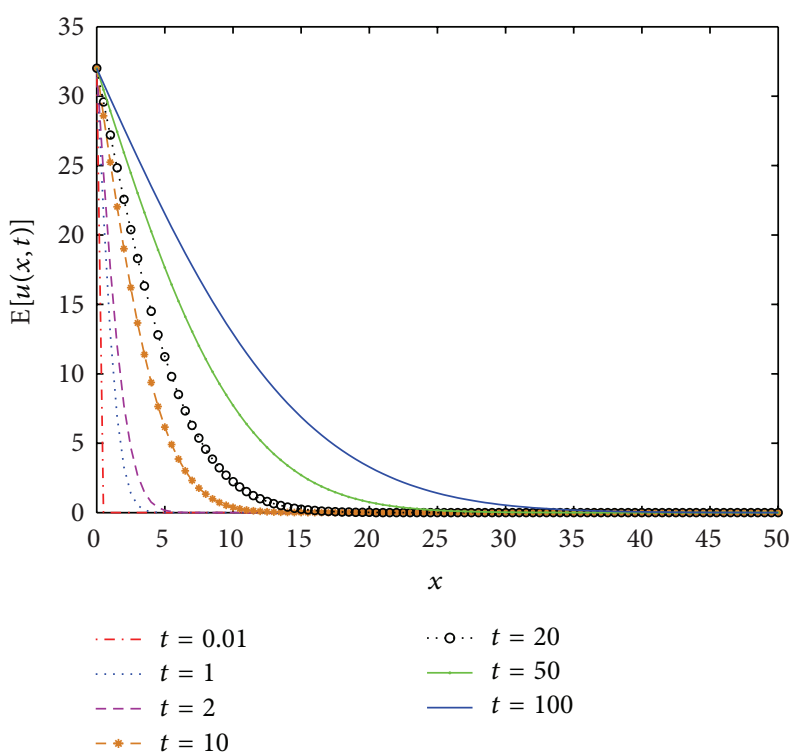

(a)

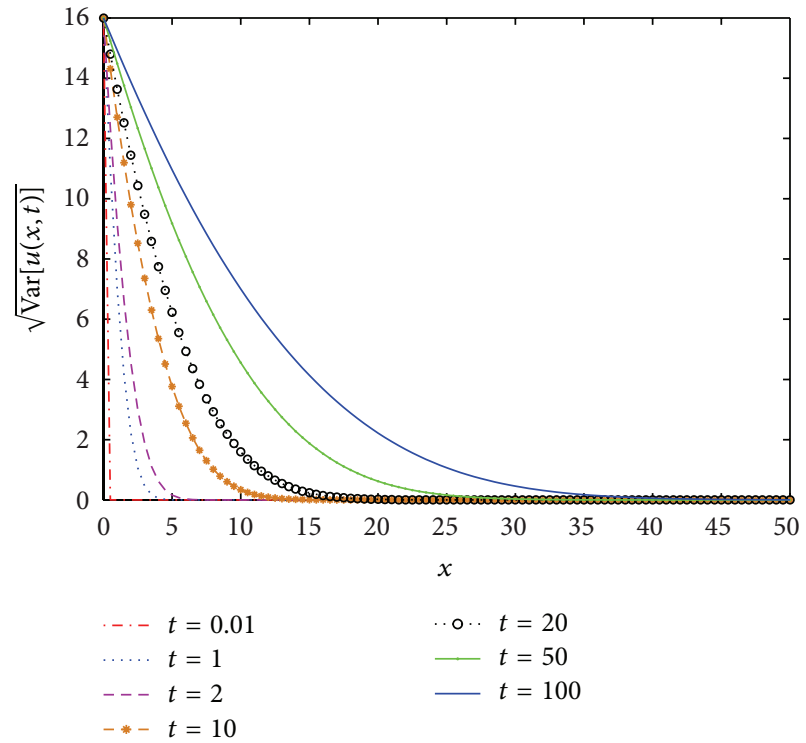

(b)

Figure 1: Approximations for the expectation $\mathrm{E}[u(x, t)](\mathrm{a})$, and the standard deviation $\sqrt{\operatorname{Var}[u(x, t)]}(\mathrm{b})$, on the spatial domain $0 \leq x \leq 50$ for some selected values in the time interval $0<t \leq 100$ in the context of Example 1 .

Theorem 5. Let one consider the random heat problem given by (65)-(67) where $L$ is a positive 4-r.v. satisfying conditions (11) and (49). Let one assume that $g(t ; B)$ is a mean fourth continuous process depending on r.v. B such that, for each $t>0$, the 4-r.v. $g(t ; B)$ is independent of L. Then, the solution stochastic process $u(x, t)$ of this problem is given by (79). In addition, (80) together with (63) and (81) are closed expressions for its expectation and variance.

\section{Numerical Examples}

Example 1. Let us consider problem (41)-(43) where the positive 4-r.v's $L$ and $A$ are assumed to follow a beta distribution of parameters $\alpha=3$ and $\beta=1: L \sim B e(3 ; 1)$ and a gamma distribution of parameters $\alpha=4$ and $\beta=8$ : $A \sim$ Gamma $(4 ; 8)$, respectively. We will assume that both r.v's are independent. Note that $L$ satisfies condition (55) since it is bounded (see Remark 1 and (11)-(13)). Furthermore, it is easy to check that the moment generating function of r.v. $L, \Phi_{L}(t)$, satisfies

$$
\Phi_{L}(t)=\mathrm{E}\left[e^{t L}\right]=3 \frac{\left(-2+2 e^{t}-2 e^{t} t+e^{t} t^{2}\right)}{t^{3}} \stackrel{t \rightarrow 0}{\longrightarrow} 1
$$

hence, it is locally bounded about $t=0$. Therefore, by Theorem 4 expression (61) is a solution s.p. of problem (41)(43). In Figure 1, we have plotted the expectation, $\mathrm{E}[u(x, t)]$, and the standard deviation, $\sqrt{\operatorname{Var}[u(x, t)]}$, of the solution s.p. on the spatial domain $0 \leq x \leq 50$ for some selected values in the time interval $0<t \leq 100$. One observes the average of temperature pulls out of zero as time increases and, as a consequence, its variability, measured through standard deviation, behaves analogously.
Example 2. Let us consider problem (65)-(67) where $L$ is assumed to follow a beta distribution of parameters $\alpha=6$ and $\beta=8: L \sim B e(6 ; 8)$. Notice that $L$ is a positive 4 -r.v. and satisfies condition (11) because it is bounded. In addition, it is straightforward to check that its moment generating function is given by

$$
\begin{aligned}
\Phi_{L}(t)=\frac{1235520}{t^{13}}( & 3991680-3991680 e^{t} \\
& +2328480 t+1663200 e^{t} t \\
& +635040 e^{2}-302400 e^{t} t^{2}+105840 t^{3} \\
& +30240 e^{t} t^{3}+11760 t^{4}-1680 e^{t} t^{4} \\
& \left.+882 t^{5}+42 e^{t} t^{5}+42 t^{6}+t^{7}\right)
\end{aligned}
$$

and satisfies $\Phi_{L}(t) \stackrel{t \rightarrow 0}{\longrightarrow} 1$; hence, it is locally bounded about $t=0$. Let us consider the boundary condition $g(t ; B)=t B$, where $B$ is a Gaussian 4-r.v. of mean $\mu=4$ and standard deviation $\sigma=0.5$, that is, $B \sim N(4 ; 0.5)$ independent of r.v. L. Since $\mathrm{E}\left[B^{4}\right]=3(0.5)^{4}<\infty$ (see [9, page 26]), $\|g(t ; B)-g(s ; B)\|_{4}=\|B\|_{4}|t-s| \stackrel{t \rightarrow s}{\longrightarrow} 0, g(t ; B)$ is m.f. continuous. Hence, the hypotheses of Theorem 5 are satisfied and expression given by (79) is a solution s.p. of problem (65)-(67). In Figure 2, we show, by means of a surface, approximations on the spatial domain $0<x \leq 8$ to the expectations and standard deviation according to (80), (63), and (81) at different instants in the time interval $1 \leq t \leq 10$. 


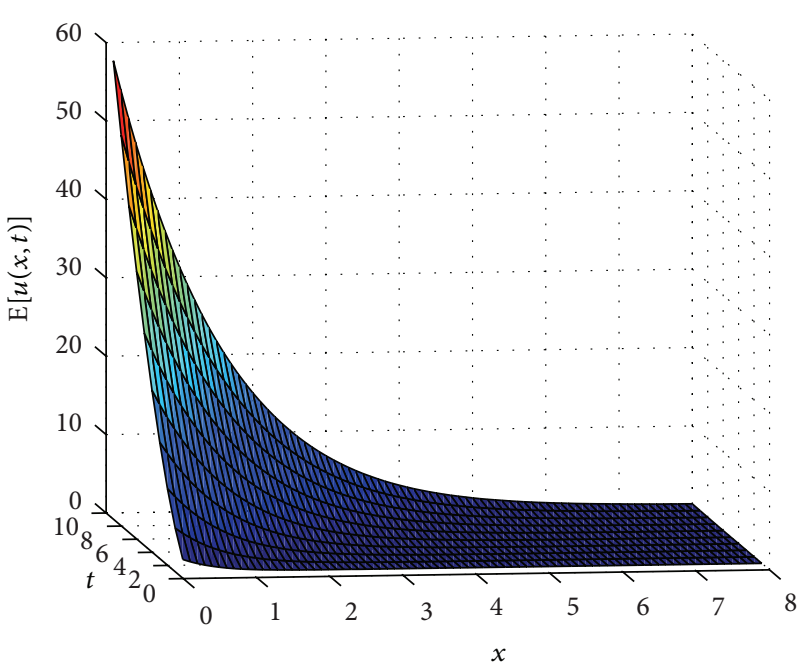

(a)

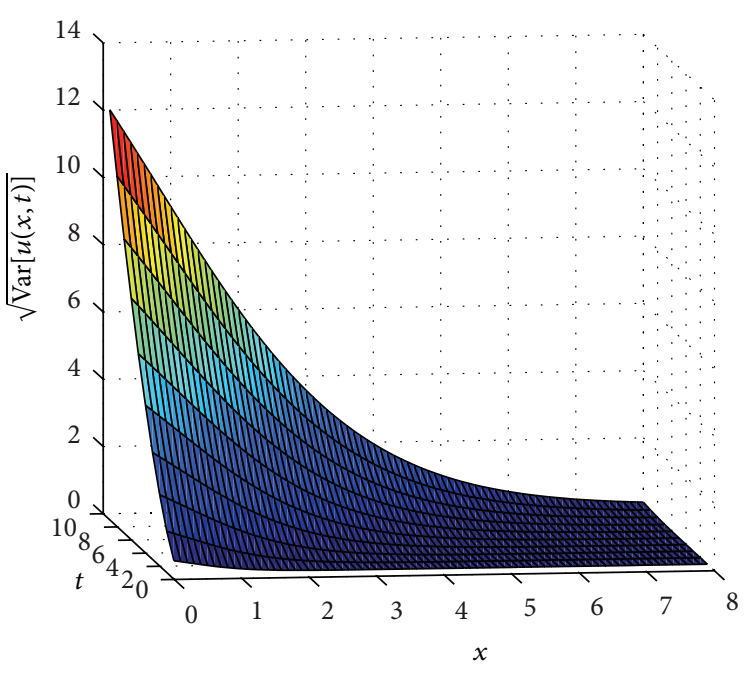

(b)

FIgURE 2: Approximations for the expectation $\mathrm{E}[u(x, t)](\mathrm{a})$, and the standard deviation $\sqrt{\operatorname{Var}[u(x, t)]}(\mathrm{b})$, on the spatial domain $0<x \leq 8$ for some values of time in the interval $1 \leq t \leq 10$ for the data of Example 2 .

\section{Conclusions}

In this paper, we show that the well-known Fourier sine and cosine transforms' technique used in the deterministic case can also be used to solve random heat problems with the same quality answer. This fact requires the proof of the m.s. operational rules for the random Fourier sine and cosine transforms, as well as results about random ordinary differential equations obtained by the authors.

Thus, the paper opens a fruitful research activity in the management of random partial differential problems not only with these random Fourier transforms but also with other random transforms.

\section{Acknowledgments}

This work has been partially supported by the Ministerio de Economía y Competitividad Grant no. DPI2010-20891-C0201 and Universitat Politècnica de València Grant no. PAID06$11-2070$

\section{References}

[1] M. Necati Özişik, Boundary Value Problems of Heat Conduction, Dover, New York, NY, USA, 1968.

[2] R. Chiba, "Stochastic heat conduction analysis of a functionally grade annular disc with spatially random heat transfer coefficients," Applied Mathematical Modelling, vol. 33, no. 1, pp. 507523, 2009.

[3] Y. Li and S. Long, "A finite element model based on statistical two-scale analysis for equivalent heat transfer parameters of composite material with random grains," Applied Mathematical Modelling, vol. 33, no. 7, pp. 3157-3165, 2009.

[4] J. C. Cortés, L. Jódar, L. Villafuerte, and R. J. Villanueva, "Computing mean square approximations of random diffusion models with source term," Mathematics and Computers in Simulation, vol. 76, no. 1-3, pp. 44-48, 2007.

[5] L. Jódar, J. C. Cortés, and L. Villafuerte, "A discrete eigenfunctions method for numerical solution of random diffusion models," in Proceedings of the Conference on Differential and Difference Equations and Applications, pp. 457-466, 2005.

[6] J.-C. Cortés, L. Jódar, M.-D. Roselló, and L. Villafuerte, "Solving initial and two-point boundary value linear random differential equations: a mean square approach," Applied Mathematics and Computation, vol. 219, no. 4, pp. 2204-2211, 2012.

[7] A. G. Madera, "Modelling of stochastic heat transfer in a solid," Applied Mathematical Modelling, vol. 17, no. 12, pp. 664-668, 1993.

[8] A. G. Madera and A. N. Sotnikov, "Method for analyzing stochastic heat transfer in a fluid flow," Applied Mathematical Modelling, vol. 20, no. 8, pp. 588-592, 1996.

[9] T. T. Soong, Random Differential Equations in Science and Engineering, Academic Press, New York, NY, USA, 1973.

[10] L. Villafuerte, C. A. Braumann, J.-C. Cortés, and L. Jódar, "Random differential operational calculus: theory and applications," Computers \& Mathematics with Applications, vol. 59, no. 1, pp. 115-125, 2010.

[11] L. Arnold, Stochastic Differential Equations: Theory and Applications, John Wiley \& Sons, New York, NY, USA, 1974.

[12] E. C. Titchmarsch, The Theory of Functions, Oxford University Press, New York, NY, USA, 2nd edition, 1939.

[13] G. Calbo, J.-C. Cortés, and L. Jódar, "Random Hermite differential equations: mean square power series solutions and statistical properties," Applied Mathematics and Computation, vol. 218, no. 7, pp. 3654-3666, 2011.

[14] M. Loève, Probability Theory I and II, vol. 45 of Graduate Texts in Mathematics, Springer, New York, NY, USA, 4th edition, 1977.

[15] J. C. Cortés, P. Sevilla-Peris, and L. Jódar, "Analytic-numerical approximating processes of diffusion equation with data uncertainty," Computers \& Mathematics with Applications, vol. 49, no. 7-8, pp. 1255-1266, 2005. 
[16] A. J. Jerri, Integral and Discrete Transforms with Applications and Error Analysis, vol. 162 of Pure and Applied Mathematics, Marcel Dekkers, New York, NY, USA, 1992.

[17] J. Dieudonné, Foundations of Modern Analysis, Academic Press, New York, NY, USA, 1969. 


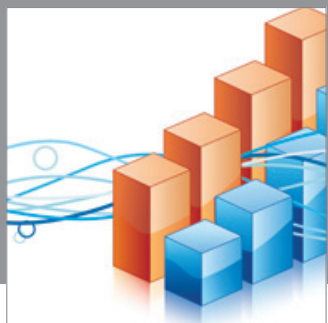

Advances in

Operations Research

mansans

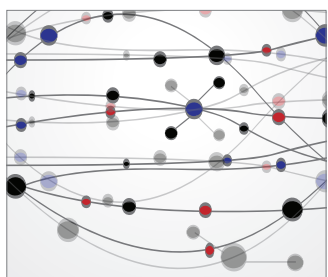

The Scientific World Journal
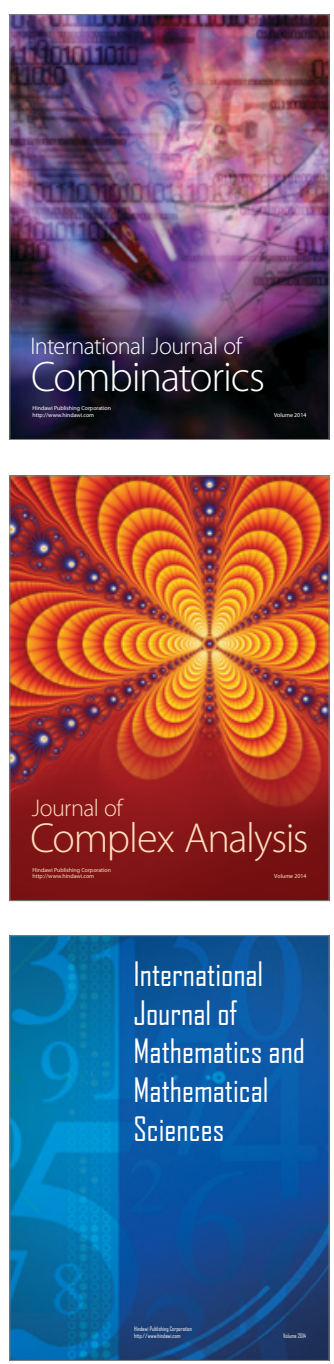
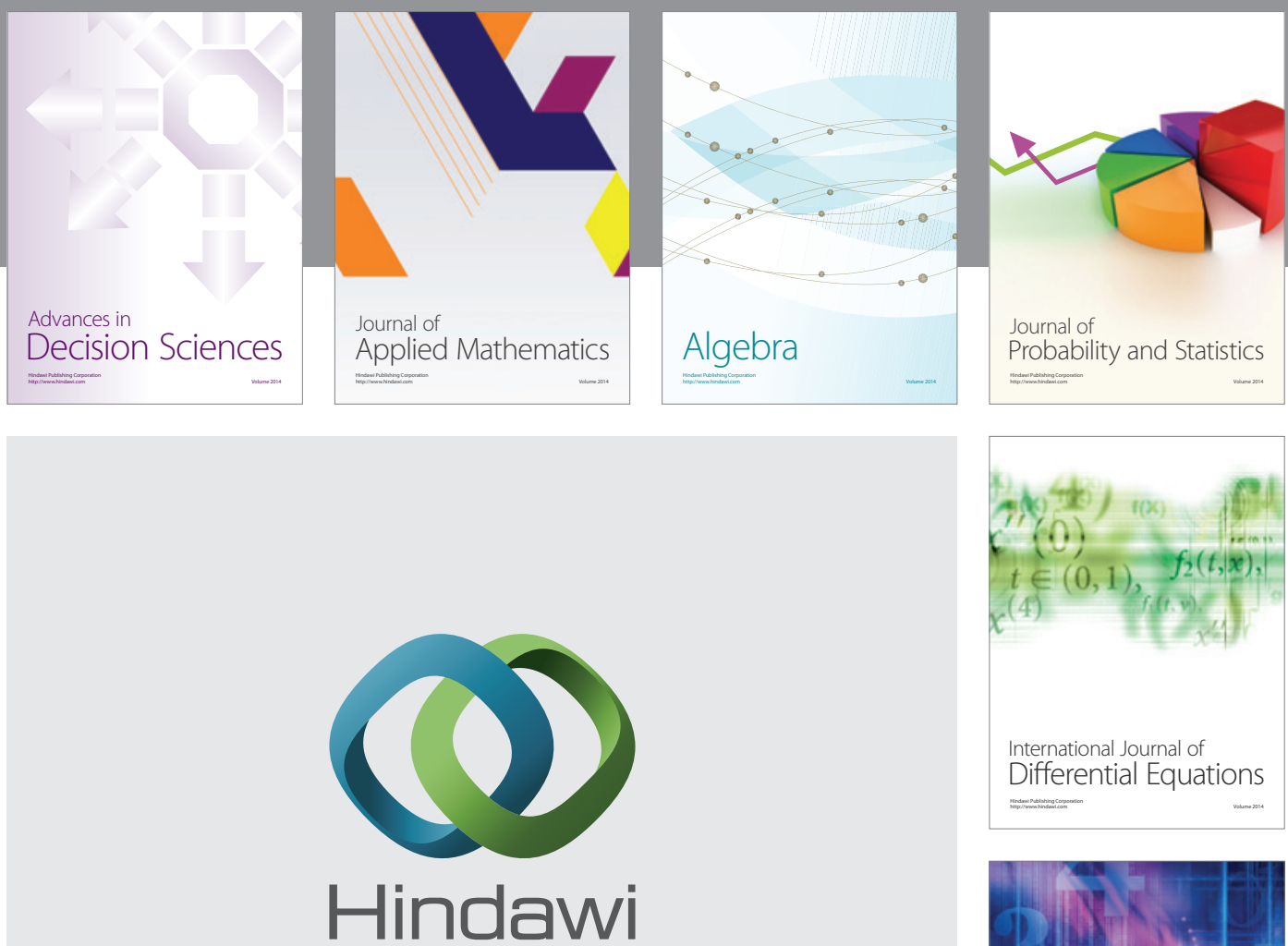

Submit your manuscripts at http://www.hindawi.com
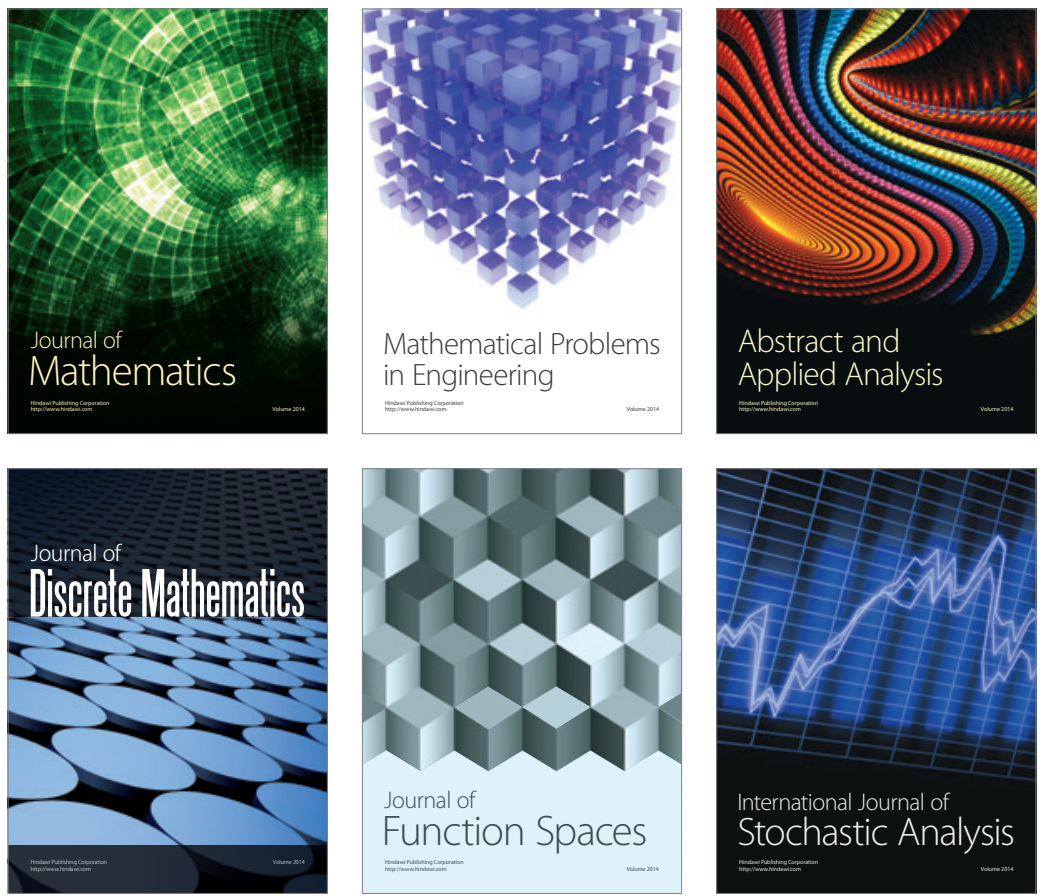

Journal of

Function Spaces

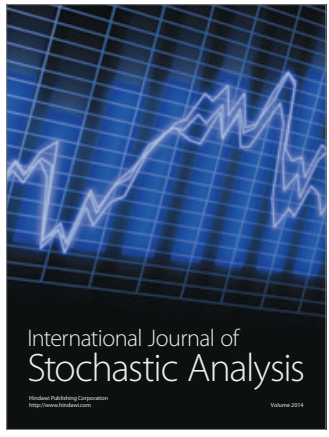


